\title{
Transference-focused psychotherapy $v$. treatment by community psychotherapists for borderline personality disorder: randomised controlled trial
}

\author{
Stephan Doering, Susanne Hörz, Michael Rentrop, Melitta Fischer-Kern, Peter Schuster, \\ Cord Benecke, Anna Buchheim, Philipp Martius and Peter Buchheim
}

\section{Background}

Transference-focused psychotherapy is a manualised treatment for borderline personality disorder.

\begin{abstract}
Aims
To compare transference-focused psychotherapy with treatment by experienced community psychotherapists.
\end{abstract}

\section{Method}

In a randomised controlled trial (NCT00714311) 104 female out-patients were treated for 1 year with either transferencefocused psychotherapy or by an experienced community psychotherapist

\section{Results}

Significantly fewer participants dropped out of the transference-focused psychotherapy group (38.5\% v. 67.3\%) and also significantly fewer attempted suicide $(d=0.8$, $P=0.009$ ). Transference-focused psychotherapy was significantly superior in the domains of borderline symptomatology ( $d=1.6, P=0.001$ ), psychosocial functioning $(d=1.0, P=0.002)$, personality organisation $(d=1.0, P=0.001)$ and psychiatric in-patient admissions $(d=0.5, P=0.001)$. Both groups improved significantly in the domains of depression and anxiety and the transference-focused psychotherapy group in general psychopathology, all without significant group differences $(d=0.3-0.5)$. Self-harming behaviour did not change in either group.

\section{Conclusions}

Transference-focused psychotherapy is more efficacious than treatment by experienced community psychotherapists in the domains of borderline symptomatology, psychosocial functioning, and personality organisation. Moreover, there is preliminary evidence for a superiority in the reduction of suicidality and need for psychiatric in-patient treatment.

\section{Declaration of interest}

None.
Transference-focused psychotherapy was developed by Otto F. Kernberg and is based on his model of borderline personality disorder. ${ }^{1,2}$ The efficacy of transference-focused psychotherapy has been evaluated in two randomised controlled trials (RCTs) to date. A 1-year $\mathrm{RCT}^{3}$ with 90 participants with borderline personality disorder compared transference-focused psychotherapy with dialectical behaviour therapy ${ }^{4}$ and psychodynamic supportive therapy. All three groups showed significant positive change in depression, anxiety, global functioning and social adjustment in a multiwave design. Transference-focused psychotherapy and dialectical behaviour therapy were associated with a significant improvement in suicidality, transference-focused psychotherapy and supportive therapy improved facets of impulsivity and only the former yielded a significant improvement in anger, irritability and verbal and direct assault. Moreover, only those individuals in the transference-focused psychotherapy group improved significantly in their reflective function and their attachment style. ${ }^{5}$ Giesen-Bloo et $a l^{6,7}$ compared transference-focused psychotherapy to schema-focused therapy ${ }^{8}$ in a 3-year RCT with 88 participants with borderline personality disorder. The transference-focused psychotherapy revealed a significantly higher drop-out rate $(51.2 \% v .26 .7 \%)$ and - despite improvements in all domains of outcome - significantly smaller treatment effects. The American Psychological Association (Division 12) evaluated transference-focused psychotherapy as having controversial research support. Thus, more research is needed before transference-focused psychotherapy can be considered to have modest or strong research support. ${ }^{9}$ The present study aims to bring clarity to the field and to determine whether transference-focused psychotherapy can be regarded as empirically supported treatment according to the American
Psychological Association (Division 12) criteria. ${ }^{10}$ This investigation examines the efficacy of transference-focused psychotherapy for borderline personality disorder in an RCT comparing those randomised to transference-focused psychotherapy with those randomised to a group treated by experienced psychotherapists in the community.

\section{Method}

\section{Study design}

The study was approved by the ethics commission of the Medical University Innsbruck, Austria, on 24 March 2004 (ID: UN1950) and was registered at Clinicaltrials.gov (NCT00714311). Participants were recruited at the out-patient units of the Departments of Psychiatry and Psychotherapy, Technical University of Munich, Germany, and the Psychoanalysis and Psychotherapy Department, Medical University Vienna, Austria. People who fulfilled the inclusion criteria were given a complete description of the study. Those who gave written informed consent were assessed by trained local research assistants. The results of the first assessments were sent to a researcher outside the two study centres who performed the randomisation. Participants were randomly assigned to either transferencefocused psychotherapy or experienced community psychotherapists. After randomisation participants were referred to a therapist; medication was registered continuously by the local administrators. Medication treatment was not standardised, its type and amount were decided on an individual basis by the individuals' psychiatrists in the community in both groups. One year after treatment started, outcome was assessed by the local research 
assistants, who were masked to the therapy delivered. The community therapists were interviewed by the local administrator to record number of sessions and frequency of supervision that took place as well as the drop-out rate and participants' reasons for terminating. In the transference-focused psychotherapy group these data were recorded by the supervisors.

\section{Participants}

Participants were screened by the local administrators at the outpatient units. Inclusion criteria were age 18-45 years, female gender, diagnosis of borderline personality disorder (DSM-IV) ${ }^{11}$ and sufficient knowledge of the German language. Exclusion criteria were diagnosis of antisocial personality disorder, schizophrenia, bipolar I and II disorder with a major depressive, manic or hypomanic episode during the previous 6 months, substance dependency (including alcohol) during the previous 6 months, organic pathology or mental retardation. After written informed consent was obtained from participants, inclusion and exclusion criteria were examined again within the baseline assessment. Antisocial personality disorder was an exclusion criterion if three or more DSM-IV criteria were met. Comorbidity with all other personality disorders and with Axis I disorders except those mentioned above were allowed as well as medication.

\section{Assessments}

Four research assistants, who were masked for the therapy delivered, conducted assessments before randomisation and after 1 year of treatment. Two performed pre- and post-tests, two pre- or post-tests only. Prior to the study they received comprehensive interview training and demonstrated satisfactory reliability for the Structured Clinical Interview for DSM (SCID-I and $-\mathrm{II})^{12,13}$ and Structured Interview for Personality Organization (STIPO) $)^{14}$ (intraclass correlation coefficients 0.72 to 0.78 ).

Two primary outcome measures - drop-out and suicide attempts - were chosen because they cover fundamental issues in the treatment of people with borderline personality disorder: individuals' adherence to the treatment while refraining from taking their own life. Suicidality was not chosen as the only primary outcome measure since data on it might not be available for those who dropped out.

\section{Primary outcome measures}

(a) Number of participants who dropped out.

(b) Suicide attempts: German version of the Cornell Interview for Suicidal and Self-Harming Behavior - Self Report (CISSB). ${ }^{15}$ The questionnaire was adapted from the Parasuicidal History Interview (PHI). ${ }^{16}$

\section{Secondary outcome measures}

(a) DSM-IV diagnostic criteria for borderline personality disorder and number of comorbid Axis I and II diagnoses: German version of the SCID-I and -II)..$^{12,13}$

(b) Psychosocial functioning: Global Assessment of Functioning (GAF) scale. ${ }^{11}$

(c) General psychopathology: German questionnaire versions of Beck Depression Inventory (BDI), ${ }^{17}$ State-Trait Anxiety Inventory $(\mathrm{STAI})^{18}$ and Brief Symptom Inventory (BSI). ${ }^{19}$

(d) Self-harming behaviour: see Primary outcome measures.

(e) Psychiatric in-patient admissions: German version of the Cornell Revised Treatment History Inventory (CRTHI). ${ }^{20}$
The questionnaire was adapted from the Treatment History Interview. $^{21}$

(f) Personality organisation: this construct assesses basic personality functions that are of importance in the regulation of the self and the relationships with others. ${ }^{1}$ Impairment of these functions is characteristic of individuals with borderline personality disorder. For the assessment of personality organisation the STIPO has been developed. ${ }^{14}$ The instrument provides an overall assessment of six levels of personality functioning from high (1) to low (6) and has been described elsewhere. $^{22,23}$

\section{Treatment and therapists}

Psychotherapy and medication were reimbursed by the German and Austrian health insurance companies at no expense to the participants. Psychotherapy was delivered in the private practices of licensed psychotherapists or at the psychotherapy out-patient units of university hospitals. The study period was 1 year; participants and therapists were instructed to maintain the treatment for at least this duration. Psychotherapies were to be continued if deemed necessary by the therapist and the participant and if paid by the insurance company. Additional non-study psychotherapy was not permitted in the transference-focused psychotherapy group.

\section{Transference-focused psychotherapy}

Transference-focused psychotherapy is a modified psychodynamic psychotherapy for people with borderline personality disorder. Two 50-minute sessions are delivered per week. Before treatment starts, a treatment contract is negotiated orally with the individual, covering general aspects like duration and payment as well as potential threats to the treatment specific to each patient (e.g. suicide attempts, drug misuse or anorectic behaviour). Based on the theoretical framework of Kernberg, ${ }^{1}$ the treatment focuses on the integration of internalised experiences of dysfunctional early relationships. For this purpose, the actual relationship between the individual and the therapist ('transference relationship') is examined as much as possible. In addition, transference-focused psychotherapy offers a hierarchy of thematic priorities to be used in every session. Limits are actively set by the therapist, if a patient's behaviour threatens their life, others' lives or the continuation of therapy. On the phenomenological level, the treatment aims at the reduction of impulsivity (e.g. aggression directed towards self or others, substance misuse, eating disorder), mood stabilisation, and the improvement of interpersonal relationships as well as occupational functioning. For this study the German translation of the treatment manual was used. ${ }^{24}$

The study therapists delivering transference-focused psychotherapy were experienced clinical psychologists or medical doctors, most of whom were psychodynamic psychotherapists. The average amount of professional experience after completion of psychoanalytic/psychodynamic training was 9.4 (s.d.=7.9) years. Since in Germany and Austria psychotherapy training is usually started 3-5 years after the last degree, the total number of years of clinical experience was considerably higher than the mean of years mentioned above. All therapists received 1-year training that covered $100 \mathrm{~h}$ of theory and $40 \mathrm{~h}$ of group supervision. Every therapist had to deliver transference-focused psychotherapy to at least one person with borderline personality disorder and had to attend group supervision for at least 1 year prior to the study. In total, 31 therapists participated in the study, 18 treated one person each, 8 treated two people, 3 treated three, 1 treated four, and 1 five people. 


\section{Treatment by experienced community psychotherapists}

These community psychotherapists were known as experienced and were particularly interested in people with borderline personality disorders. All of them had received referrals of these individuals by the local administrators before the study. However, none of the therapists had specific training in manualised borderline psychotherapy. The average amount of professional experience after completion of psychotherapy training was 8.9 (s.d. $=9.8$ ) years. No significant differences were found between the two groups of therapists regarding their level of experience. Individual therapy by experienced community psychotherapists was delivered according to the mental healthcare situation in Munich and Vienna. All therapists were licensed psychotherapists who were paid by the health insurance companies. In total, 19 people were treated by psychoanalysts $(36.5 \%), 18$ by behaviour therapists $(34.6 \%), 4$ by client-centered therapists (Rogerian) (7.7\%), 4 by systemic psychotherapists $(7.7 \%), 1$ by a Gestalt therapist $(1.9 \%)$. Four people $(7.7 \%)$ dropped out of the study after randomisation before being assigned to a therapist. Overall, 36 therapists participated in the study, 29 treated one person each, 5 treated two, 1 treated four and 1 five people. Within the 1-year time frame, therapists were free to choose the frequency of sessions according to their method.

\section{Supervision}

Supervision is a crucial part of transference-focused psychotherapy. Video recordings of all sessions were performed and used in the group supervision. Four supervision groups took place regularly during the whole study period either weekly for $2 \mathrm{~h}$ or every fortnight for $4 \mathrm{~h}$. Every case was supervised at least every 4-6 weeks. All supervisors were trained and certified in transference-focused psychotherapy and the supervising process at the Personality Disorders Institute of Cornell University, New York (Otto F. Kernberg). Experienced community psychotherapists attended supervision according to their usual routine; their sessions were not recorded. For this reason, transferencefocused psychotherapy psychotherapists received significantly $(P=0.003)$ more supervision $(21.5$, s.d. $=8.5$ sessions $)$ than experienced community psychotherapists ( 11.4 , s.d. $=12.3$ sessions $)$.

\section{Treatment integrity}

For the assessment of the transference-focused psychotherapy psychotherapists' treatment integrity a German translation of a specific rating of adherence and competence ${ }^{24,25}$ was used. The instrument contains ten items that are judged on a five-point scale, ranging from 1 representing low quality to 5 representing high quality of the session. Nine items address specific aspects of adherence to the manual and the tenth item addresses the overall competence the therapist exhibits in the session. The rating was performed by the supervisor after every video-guided supervision of a therapy session. If a score of 2 and below was given on the adherence and competence ratings, additional individual supervision was delivered. For the group analyses of adherence and competence, four sessions of every single therapy were included: contract setting; after 4 months of therapy; after 8 months of therapy; and after almost 12 months of therapy. The mean overall competence rating was 3.0 (s.d.=0.9). Ratings of 2 and below stand for insufficient adherence and competence, thus, a mean rating of 3.0 can be regarded as satisfactory. No comparable ratings were performed in the experienced community psychotherapists.

Since the experienced community psychotherapists were not expected to deliver a specific treatment, but rather to represent treatment as usual in the field as it is performed by psychotherapists who are interested and experienced in treating people with borderline personality disorder, no integrity checks were performed in this group.

\section{Statistical analyses}

For sample size calculation (continuity corrected) a 1-year prevalence of $30 \%$ suicide attempters among individuals with borderline personality disorder was assumed. ${ }^{6,26}$ To detect a difference between a reduction of $10 \%$ in the transference-focused psychotherapy group and of $20 \%$ in the experienced community psychotherapists group with a power of $80 \%(P<0.05), 41$ participants per group were needed.

Intention-to-treat group comparisons were calculated for the outcome analyses. Chi-squared statistics were employed for dichotomous variables, the McNemar test for within-group changes of dichotomous variables, Mann-Whitney $U$-tests for ordinal data, and $t$-tests for continuous data. Cohen's $d$ was computed to measure effect sizes for continuous data, and for dichotomous measures effect size $\left(d^{*}\right)$ was calculated according to Hasselblad \& Hedges. ${ }^{27}$ To compare the time effects of the two interventions, analyses of covariance (ANCOVA) were performed. For the group comparison of change over time of presence or absence of suicide attempts and self-harming acts during the treatment year (dichotomouos variables) additional variables were created: 1 , positive change; 0 , no change; -1 , negative change. Group differences on these variables were tested by means of the Mann-Whitney $U$-test. For the primary outcome variables (drop out, suicide attempt during the treatment year) Bonferroni adjustment was performed, which set the level of significance to $P<0.025$. $P$ for secondary outcome measures are explorative. The SPSS 15.0 software package for Windows was used for statistical analyses.

\section{Treatment of missing data}

As presented in Fig. 1, a number of participants were lost to the follow-up evaluation. Since this study contains two assessments only, we decided to address the problem of missing values by combining two strategies of analysis: observed cases and last observation carried forward (LOCF). The rationale for this combination is the fact that the latter tends to underestimate within-group changes and the former to overestimate these changes. ${ }^{28}$ To prevent unrealistically high effect sizes because of missing values on the one hand, and to rule out a bias due to different drop-out rates in the two groups on the other hand, the complete intention-to-treat analysis was performed twice. The first analysis contained only participants who attended the 1-year follow-up, and in the case of the second analysis, missing data were replaced by the baseline assessment.

\section{Results}

\section{Participant characteristics at baseline}

Participants were recruited between October 2004 and August 2006. The participant flow is presented in Fig. 1. In total, 104 women with borderline personality disorder gave written informed consent and were included in the study. Their characteristics are shown in online Table DS1. There were no significant differences between the groups with regard to sociodemographic and clinical variables at baseline. 


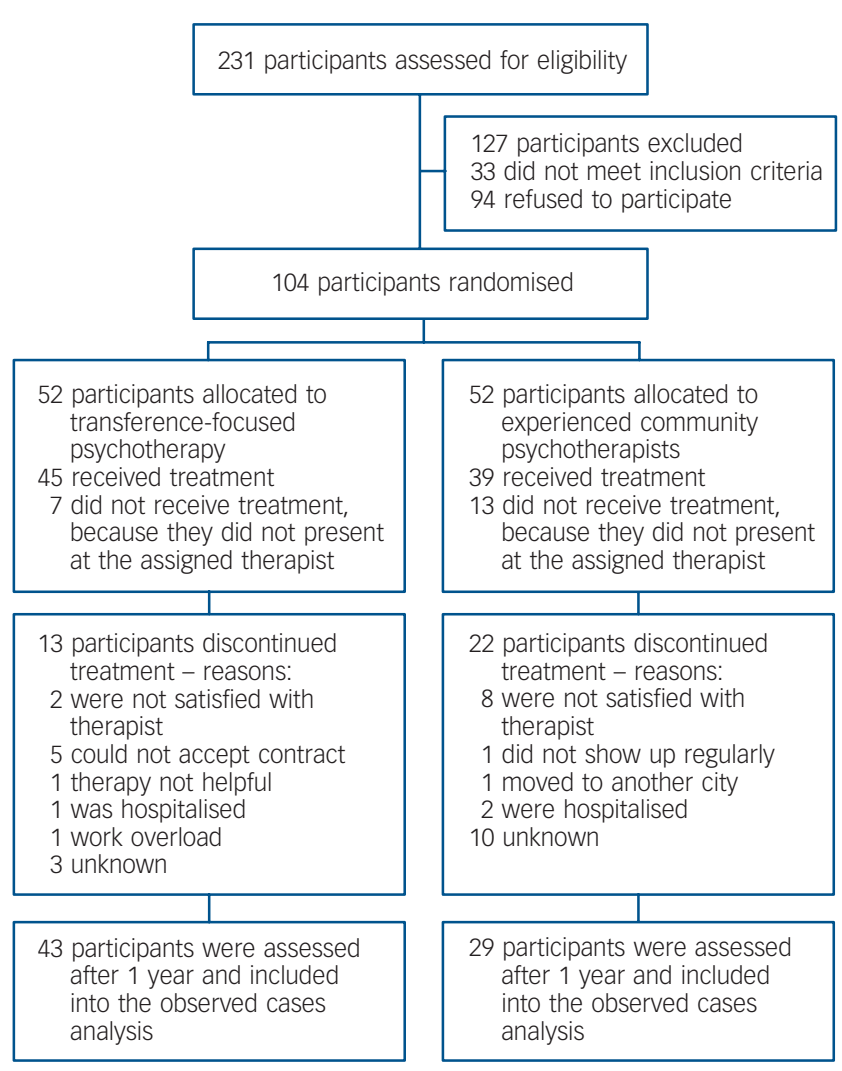

Fig. 1 Participant flow in the randomised controlled trial.

\section{Treatment received}

The drop-out rate was significantly higher (Bonferroni adjusted) in the experienced community psychotherapists group $(67.3 \% v$. $38.5 \%, \chi^{2}=8.683$, d.f. $\left.=1, P=0.003\right)$. The point of time when individuals dropped out of therapy can be seen from the Kaplan-Meier survival curve in Fig. 2 (log-rank statistic 8.453, d.f. $=1, P=0.004)$. In total, 72 participants were included into the observed cases analyses (43 in the transference-focused psychotherapy group and 29 in the experienced community psychotherapist group); the LOCF analyses contained the whole sample.

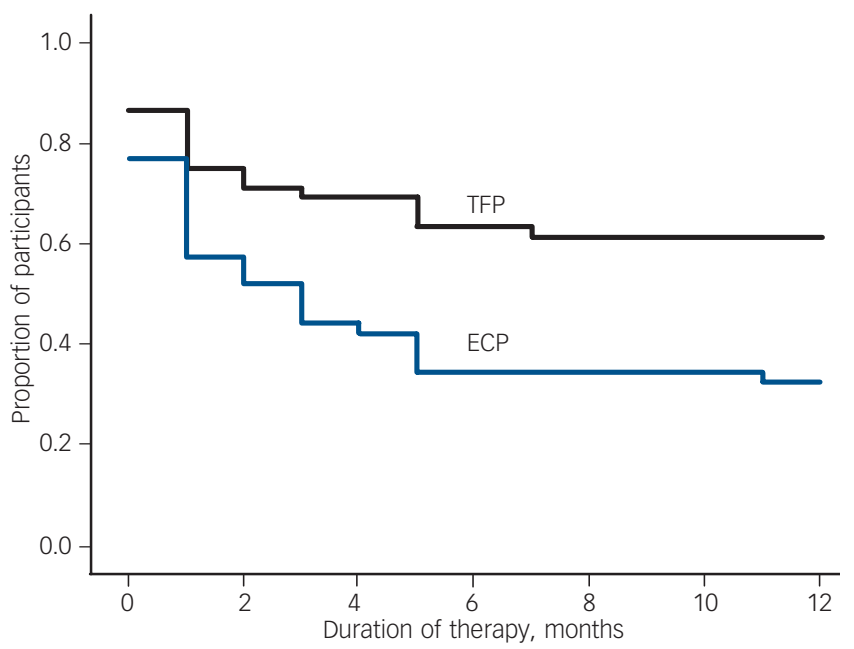

Fig. 2 Proportion of participants remaining in transferencefocused psychotherapy (TFP) and treatment by experienced community psychotherapists (ECP).
Participants in the transference-focused psychotherapy group received 48.5 (s.d. $=34.2$ ) sessions and those in the experienced community psychotherapists group $18.6($ s.d. $=24.0)$ sessions of individual psychotherapy within the 1-year study period. The difference is significant $(t=5.163$, d.f. $=101, P<0.001)$. Among the completers, transference-focused psychotherapy participants $(n=32)$ received $72.9($ s.d. $=14.5)$ sessions, and the experienced community psychotherapists group $(n=17) 39.4 \quad($ s.d. $=26.3)$ sessions, which also differs significantly $(t=4.984$, d.f. $=48$, $P<0.001)$.

\section{Medication}

There were no significant differences between the groups with regard to medication at baseline and during the 1-year treatment period (Fig. 3). The only participant who received amphetamines was in the transference-focused psychotherapy group. There was no significant influence of psychotropic medication on the outcome variables with the exception of a worse BSI global severity index in medicated participants $(F=43.927$, d.f. $=1,101$, $P=0.04)$.

\section{Treatment outcomes}

\section{Last observation carried forward analyses}

The results of the LOCF analyses are shown in online Table DS2. The transference-focused psychotherapy group showed a significantly higher proportion of participants that fulfilled less than five DSM-IV diagnostic borderline criteria after 1 year and were not diagnosed borderline personality disorder any more $\left(42.3 \%\right.$ v. $15.4 \%, \chi^{2}=9.182$, d.f. $\left.=1, P=0.002\right)$. The transferencefocused psychotherapy group was significantly superior with regard to the number of DSM-IV diagnostic criteria, psychosocial functioning, personality organisation, suicide attempts and number and duration of psychiatric in-patient treatments.

Overall, 42 participants continued psychotherapy after the 1-year study period (29 in the transference-focused psychotherapy group and 13 in the experienced community psychotherapist

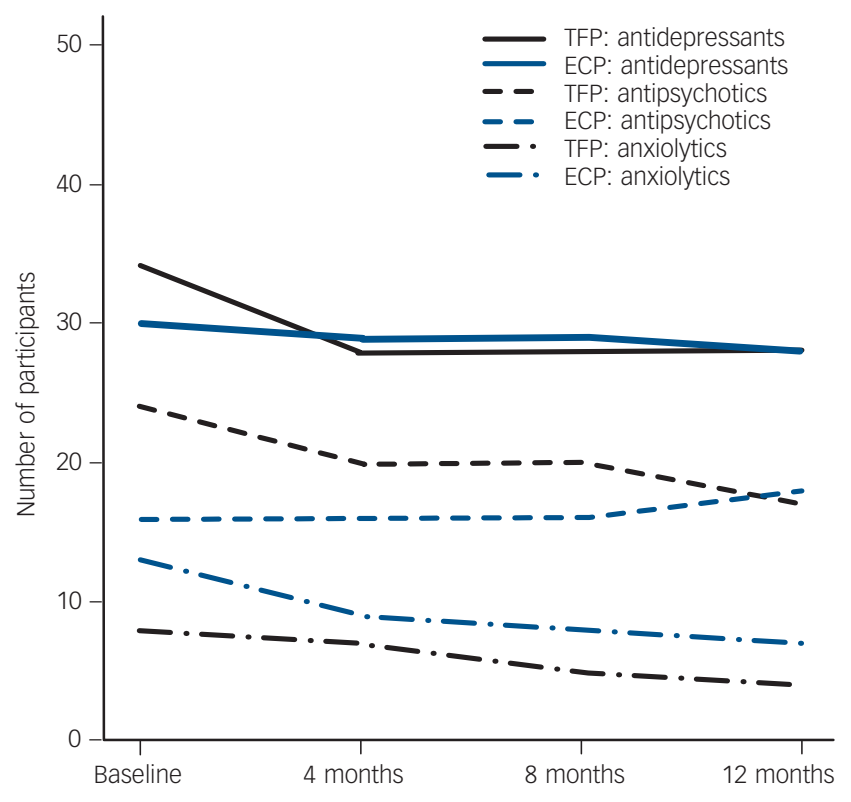

Fig. 3 Proportion of participants taking psychotropic medication. TFP, transference-focused psychotherapy; $\mathrm{ECP}$, experienced community psychotherapists. 
group). When controlled for continuation of treatment after 1 year the results for the LOCF analyses remained almost unchanged with the exception of 'suicide attempts'. Since in each group only one participant who continued therapy attempted suicide during the treatment period, the group time interaction was no longer significant here. The group time interaction remained significant for the number of psychiatric hospitalisations $(F=8.814$, d.f. $=99, P=0.004)$, but only showed a trend towards significance for the number of days in psychiatric in-patient treatment $(F=2.889$, d.f. $=99, P=0.09)$. Compared with the whole sample, participants who continued therapy showed equal or even higher effect sizes. The latter was the case for the GAF score $(d=1.4)$ and DSM-IV borderline criteria $(d=1.9)$ in the transference-focused psychotherapy group, and for depression $(d=0.9)$ in the experienced community psychotherapists group.

\section{Observed cases analyses}

The observed cases analyses revealed similar results to the LOCF with higher effect sizes and somewhat lower significance. In the transference-focused psychotherapy group, significantly more participants had less than five DSM-IV borderline criteria after 1 year $\left(51.2 \%\right.$ v. $27.6 \%, \chi^{2}=3.961$, d.f. $\left.=1, P=0.047\right)$ and a tendency towards a greater reduction in these $(F=3.986$, d.f. $=1,69, P=0.05, d=1.8 \quad v . d=1.4)$, a significantly greater improvement in psychosocial functioning $(F=4.765$, d.f. $=1,69$, $P=0.03, \quad d=1.1 \quad v . \quad d=0.5), \quad$ and a significantly greater improvement in personality organisation $(F=6.420$, d.f. $=1,69$, $P=0.02, d=1.2$ v. $d=0.4$ ). Moreover, participants in the transference-focused psychotherapy group improved significantly with regard to suicide attempts $\left(\right.$ McNemar's $\chi^{2}=13.09$, d.f. $=1$, $P=0.001)$ and number of suicide attempts $(t=2.991$, d.f. $=36$, $P=0.005)$, whereas those in the experienced community psychotherapists group remained unchanged; the group comparison was significant for number of suicide attempts $(F=5.125$, d.f. $=1,55, P=0.03)$. Participants in the transferencefocused psychotherapy group reported significantly fewer psychiatric in-patient admissions $(t=3.333$, d.f. $=25, P=0.003$, $d=0.5)$ of shorter duration ( $t=2.620$, d.f. $=21, P=0.02, d=0.6$, whereas this did not change in the other group; the group comparison did not reach significance. Both groups did not show a significant change in self-harming behaviour. Significant improvement in self-reported psychopathology occurred in all dimensions with no significant group differences $(d=0.3-0.8)$.

\section{Control for dose effect}

Participants treated with transference-focused psychotherapy received more psychotherapy sessions than those in the experienced community psychotherapists group. This was also the case among the completers. To rule out a mere dose effect of transference-focused psychotherapy, completer analyses were conducted, controlling for the number of therapy sessions delivered. The group differences remained significant for GAF score $(F=4.639$, d.f. $=1,45, P=0.04, d=1.5$ v. 0.6), number of DSM-IV borderline criteria $(F=5.168$, d.f. $=1,45, \quad P=0.03$, $d=1.9 v .1 .2)$, and level of personality organisation $(F=4.168$, d.f. $=1,45, P<0.05, d=1.0 v$. 0.4). In both groups all but one of the individuals who attempted suicide dropped out of treatment (6 in the transference-focused psychotherapy group and 10 in the experienced community psychotherapists group), and a considerable number after psychiatric in-patient admissions (9 in the transference-focused psychotherapy group and 18 in the experienced community psychotherapists group). Those individuals who dropped out were not included in the completer analyses. As a consequence, the group differences with regard to suicide attempts and psychiatric in-patient admissions decreased below the level of significance.

\section{Discussion}

This study investigated the efficacy of transference-focused psychotherapy compared with treatment by experienced community psychotherapists for people with borderline personality disorder. The results demonstrate the significant superiority of transference-focused psychotherapy with regard to the primary outcome criteria of drop-out rate and suicide attempts during the treatment year. The same was true for the secondary outcome criteria reduction of DSM-IV diagnostic borderline criteria, psychosocial functioning, level of personality organisation and psychiatric in-patient admissions.

\section{Selection of participants}

This study involved people with less severe borderline personality disorder, with higher GAF scores, fewer comorbid Axis I and II disorders and fewer self-harming acts than other treatment studies on this patient group. This can be attributed to the healthcare situation in Germany and Austria, where more severely ill people with borderline personality disorder receive in-patient treatment paid for by healthcare insurance companies. Moreover, people with comorbid antisocial personality disorder and substance dependency were excluded. This patient selection limits the generalisability of the results.

\section{Drop-out rates}

The drop-out rate of participants in the transference-focused psychotherapy group $(38.5 \%)$ was somewhat higher than that reported for out-patient dialectic behavioural therapy $(25 \%)^{29}$ and schema-focused therapy $(26.7 \%){ }^{6}$ This can be attributed to the healthcare situation in Germany and Austria, where insurance companies cover psychotherapy for everyone in need. As a consequence, the study participants received psychotherapy at no cost to them and were free to choose another therapist if they were not satisfied with the one they were assigned to. Individuals who do not receive manualised borderline-specific psychotherapy drop out of therapy much more often. Linehan et al ${ }^{29}$ reported a $59.2 \%$ drop-out rate in their community treatment by experts group; which is close to the $67.3 \%$ in our study.

\section{Suicidality and self-injury}

Participants in the transference-focused psychotherapy group had improved suicidality but self-injury was not reduced, whereas in the comparison group both were almost unchanged; this calls for further interpretation, since other treatment approaches reduce both suicidality and self-injury. ${ }^{6,29,30}$ A possible explanation for this difference may be found in the study by Verheul et $a l^{31}$ who reported an improvement in self-injury after 1 year of dialectic behavioral therapy only in individuals with a high severity of these behaviours, whereas individuals with a low severity remained unchanged. In our sample, the median number of self-injurious acts in the year before treatment was 6.0 (range $0-365)$; Linehan et $a l^{29}$ reported a median of 10 acts during the pre-treatment year and Bateman \& Fonagy ${ }^{30}$ in their paper on partially hospitalised patients reported $8-9$ self-injuries in the 6 months prior to treatment. Thus, our participants revealed a comparably low severity of self-injurious behaviour, which according to the finding of Verheul et $a l^{31}$ - might have led to the lack of positive change. 


\section{Depression, anxiety and general psychopathology}

Transference-focused psychotherapy yielded lower effect sizes in its improvement in self-reported depression, anxiety and general psychopathology, and as a result no significant differences between groups were found. This finding is in line with previous RCTs that also did not find significant group differences with regard to depression and anxiety in individuals treated with behavioural therapy $^{3,29,31,32}$ and transference-focused psychotherapy. ${ }^{3}$ In contrast, Bateman \& Fonagy ${ }^{30}$ reported highly significant group differences in the improvement of depression and anxiety in participants with borderline personality disorder treated with mentalisation-based therapy, ${ }^{33}$ but their participants were very severely ill - they started with a BDI score of 36.0 (compared with 25.8 in our transference-focused psychotherapy group), received intense 18-month partial hospitalisation treatment and were compared with a group of participants who only received two psychiatric consultations per month.

\section{Dose-effect relationship}

Since completed therapies with experienced community psychotherapists included significantly fewer sessions than completed transference-focused psychotherapies, completer analyses were conducted, controlling for the effect of number of sessions. The results showed that the effect of transference-focused psychotherapy can not be solely reduced to the dose of treatment. The fact that the group differences with regard to suicide attempts and psychiatric admissions were no longer significant can easily be explained by the high number of people who dropped out after suicide attempts and/or hospitalisation, especially in the experienced community psychotherapists group.

\section{Continuation of therapy after the study period}

In our study a number of participants continued psychotherapy after the 1-year study period. This has also been the case in previous influential studies ${ }^{6,34}$ and has to be regarded as a potential bias to the results. As a consequence we controlled the outcome analyses for continuation of therapy and found the main effects to be stable. Since in both groups only one of the participants who continued treatment attempted suicide, the group time interaction for suicide attempts during the study period was no longer significant. As mentioned above, the rest of the participants who attempted suicide during the study period (six in the transference-focused psychotherapy group and ten in the experienced community psychotherapists group) dropped out from therapy before the end of the 1-year study period.

\section{Strengths and limitations}

The strengths of this study are its large sample size, the multisite setting, the treatment by experienced community psychotherapists in the comparison group and the conservative statistical evaluation including observed cases and LOCF analyses. Due to the earlier mentioned healthcare situation in Germany and Austria, which allows individuals to switch therapies at no cost and to abstain from the study follow-up assessments without losing their therapy, there are two major limitations of the study: the high drop-out rate and the low participation in the follow-up assessment. Only 49 participants $(47.1 \%)$ in the whole sample completed the 1-year treatment phase, end-point data were available for 71 individuals $(68.3 \%)$. This amount of missing data reduces the validity of the results to a certain degree. Completer analyses and control of the results for dose-effect relationship assured that despite the missing data a genuine and significant efficacy of transference-focused psychotherapy exists in the domains of borderline symptomatology, psychosocial functioning and personality organisation. Evidence for a superiority in the reduction of suicidality and need for psychiatric in-patient treatment should be regarded as preliminary.

\section{Implications of the study}

This study strengthens the empirical validation of transferencefocused psychotherapy for the treatment of people with borderline personality disorder. Future research should look at long-term follow-up, since effects of psychotherapy seem to take years to develop and to continue after termination of treatment. ${ }^{35}$ Moreover, given that we now have dialectic behavioural treatment, transference-focused psychotherapy, mentalisation-based therapy and schema-focused therapy as viable treatments for borderline personality disorder, the specific and potentially different mechanisms of change need to be studied in order to determine which treatment is most effective for which individuals with specific problems.

Stephan Doering, MD, Department of Prosthodontics and Material Sciences, Department of Psychosomatics and Psychotherapy, University of Muenster, Germany, and Department of Medical Psychology and Psychotherapy, Medical University Innsbruck, Austria; Susanne Hörz, PhD, Department of Psychiatry and Psychotherapy, Technical University Munich and Department of Psychology, University of Munich, Germany; Michael Rentrop, MD, Department of Psychiatry and Psychotherapy, Technical University Munich, Germany; Melitta Fischer-Kern MD, Peter Schuster, MD, Department of Psychoanalysis and Psychotherapy, Medical University of Vienna, Austria; Cord Benecke, PhD, Anna Buchheim, PhD, Department of Clinical Psychology, University of Innsbruck, Austria; Philipp Martius MD, Department of Psychiatry and Psychotherapy, Technical University Munich, and Klinik Höhenried gGmbH, Bernried, Germany; Peter Buchheim, MD, Department of Psychiatry and Psychotherapy, Technical University Munich, Germany

Correspondence: Stephan Doering, MD, Psychosomatics in Dentistry, Department of Prosthodontics and Material Sciences, University of Muenster, Waldeyerstrasse 3048149 Muenster, Germany. Email: Stephan.doering@ ukmuenster.de

First received 29 Jun 2009, final revision 15 Jan 2010, accepted 22 Jan 2010

\section{Funding}

Supported by grant number 10636 from the Jubiläumsfonds of the Austrian National Bank.

\section{Acknowledgements}

The authors thank Angelika Binder-Krieglstein, MA, Nora Frossard, MD, and Nestor Kapusta, MD, for data collection, Professor Hans Förstl, Department of Psychiatry and Psychotherapy, Technical University of Munich, Germany, and Professor Marianne SpringerKremser, Department of Psychoanalysis and Psychotherapy, Medical University Vienna, Austria, for institutional support.

\section{References}

1 Kernberg OF. Severe Personality Disorders. Yale University Press, 1984.

2 Clarkin JF, Yeomans FE, Kernberg OF. Psychotherapy for Borderline Personality. Focusing on object Relations. American Psychiatric Publishing, 2006.

3 Clarkin JF, Levy KN, Lenzenweger MF, Kernberg OF. Evaluating three treatments for borderline personality disorder: a multiwave study. Am J Psychiatry 2007; 164: 1-8.

4 Linehan MM. Cognitive-Behavioral Treatment of Borderline Personality Disorder. Guilford Press, 1993.

5 Levy KN, Meehan KB, Kelly KM, Reynoso JS, Weber M, Clarkin JF, et al. Change in attachment patterns and reflective function in a randomized control trial of transference-focused psychotherapy for borderline personality disorder. J Consult Clin Psychol 2006; 74: 1027-40.

6 Giesen-Bloo J, van Dyck R, Spinhoven P, van Tilburg W, Dirksen C, van Asselt T, et al. Outpatient psychotherapy for borderline personality disorder. Randomized trial of schema-focused therapy vs transference-focused psychotherapy. Arch Gen Psychiatry 2006; 63: 649-58. 
7 van Asselt ADI, Dirksen CD, Arntz A, Giesen-Bloo JH, van Dyck R, Spinhoven P, et al. Out-patient psychotherapy for borderline personality disorder: costeffectiveness of schema-focused therapy $v$. transference-focused psychotherapy. Br J Psychiatry 2008; 192: 450-7.

8 Young JE. Cognitive Therapy for Personality Disorders: A Schema-Focused Approach (3rd edn). Professional Resource Press, 1999.

9 Society of Clinical Psychology, American Psychological Association, Division 12. Transference-Focused Therapy for Borderline Personality Disorder. APA 2009 (http://www.psychology.sunysb.edu/eklonsky-/division12/treatments/ bpd_transference.html).

10 Chambless DL, Baker MJ, Baucom DH, Beutler LE, Calhoun KS Crits-Christoph $\mathrm{P}$, et al. Update on empirically validated therapies, II. Clin Psychol 1998; 51: 3-16.

11 American Psychiatric Association. Diagnostic and Statistical Manual of Mental Disorders (4th edn) (DSM-IV). APA, 1994.

12 Wittchen HU, Zaudig M, Fydrich T. SKID-I: Strukturiertes Klinisches Interview für DSM-IV, Achse I [SCID-I: Structured Clinical Interview for DSM-IV, Axis I] Hogrefe, 1997.

13 Fydrich T, Renneberg B, Schmitz B, Wittchen HU. SKID-II: Strukturiertes Klinisches Interview für DSM-IV, Achse II: Persönlichkeitsstörungen [SCID-II: Structured Clinical Interview for DSM-IV, Axis II: Personality disorders]. Hogrefe, 1997.

14 Clarkin JF, Caligor E, Stern B, Kernberg OF. Structured Interview of Personality Organization (STIPO). Weill Medical College of Cornell University, 2004.

15 Clarkin JF. Cornell Interview for Suicidal and Self-Harming Behavior - Self Report (CISSB). Weill Medical College of Cornell University, 1998

16 Linehan MM, Wagner AW, Cox G. Parasuicide History Interview: Comprehensive Assessment of Parasuicidal Behavior. University of Washington, 1989.

17 Hautzinger M, Bailer M, Worall H, Keller F. Beck-Depressions-Inventar (BDI) [Beck Depression Inventory]. Huber, 1994.

18 Laux L, Glanzmann P, Schaffner P, Spielberger CD. Das State-Trait-AngstInventar (STAI). Theoretische Grundlagen und Handanweisung [The State-Trait Anxiety Inventory. Theoretical Background and Instruction Manual]. Beltz, 1984.

19 Franke GH. Brief Symptom Inventory von L.R. Derogatis (Kurzform der SCL-90-R) - Deutsche Version. Beltz, 2000

20 Clarkin JF. Cornell Revised Treatment History Inventory (CRITH). Weill Medical College of Cornell University, 1998.

21 Linehan MM. Treatment History Interview (THI). University of Washington, 1987.
22 Stern BL, Caligor E, Clarkin JF, Critchfield KL, Hörz S, Maccornack V, et al. Structured interview of personality organization (STIPO): preliminary psychometrics in a clinical sample. J Pers Assess 2010; 92: 35-44.

23 Hörz S. A Prototype of Borderline Personality Organization Assessed by the Structured Interview of Personality Organization (STIPO). Kovac, 2007.

24 Clarkin JF, Yeomans FE, Kernberg OF. Psychotherapie der BorderlinePersönlichkeit. Manual zur psychodynamischen Therapie [Psychotherapy for Borderline Personality. Manual of Psychodynamic Treatment]. Schattauer, 2001.

25 Yeomans F, Hull J, Delaney J, Clarkin JF. An Instrument For Rating Therapist Adherence To A Psychodynamic Treatment Model. Weill Medical College of Cornell University, 2004.

26 Gunderson JG, Ridolfi ME. Borderline personality disorder. suicidality and selfmutilation. Ann N Y Acad Sci 2001; 932: 61-73.

27 Hasselblad V, Hedges LV. Meta-analysis of screening and diagnostic tests. Psychol Bull 1995; 117: 167-78.

28 Prakash A, Risser RC, Mallinckrodt $\mathrm{CH}$. The impact of analytic method on interpretation of outcomes in longitudinal clinical trials. Int J Clin Pract 2008; 62: 1147-58.

29 Linehan MM, Comtois KA, Murray AM, Brown MZ, Gallop RJ, Heard HL, et al. Two-year randomized controlled trial and follow-up of dialectical behavior therapy vs therapy by experts for suicidal behavior and borderline personality disorder. Arch Gen Psychiatry 2006; 63: 757-66.

30 Bateman A, Fonagy P. Effectiveness of partial hospitalization in the treatment of borderline personality disorder: a randomized controlled trial. Am J Psychiatry 1999; 156: 1563-9.

31 Verheul R, van den Bosch LMC, Koeter MWJ, de Ridder MAJ, Stijnen T, van den Brink W. Dialectical behavior therapy for women with borderline personality disorder: 12-month, randomised clinical trial in The Netherlands. Br J Psychiatry 2003; 182: 135-40.

32 Linehan MM, Armstrong HE, Suarez A, Allmon D, Heard HL. Cognitivebehavioral treatment of chronically parasuicidal borderline patients. Arch Gen Psychiatry 1991; 48: 1060-4.

33 Bateman A, Fonagy A. Psychotherapy for Borderline Personality Disorder Mentalization-Based Treatment. Oxford University Press, 2004.

34 Bateman A, Fonagy P. Treatment of borderline personality disorder with psychoanalytically oriented partial hospitalization: an 18 month follow-up. Am J Psychiatry 2001; 158: 36-42.

35 Levy KN. Psychotherapies and lasting change. Am J Psychiatry 2008; 165 : $556-9$. 